\title{
Anatomical variations in human carotid bodies
}

\author{
Q KHAN, D HEATH, P SMITH \\ From the Department of Pathology, University of Liverpool, Liverpool
}

SUMMARY The variations in anatomical structure and position of both carotid bodies were noted in 100 consecutive subjects who came to necropsy. Considerable variations in form were found. Although most carotid bodies (83\% on the right and $86 \%$ on the left) were of the classic ovoid type, an appreciable minority was bilobed $(9 \%$ on the right and $7 \%$ on the left) or double $(7 \%$ on the right and $6 \%$ on the left); $1 \%$ were leaf shaped. All these anatomical variants have to be distinguished from the pathologically enlarged carotid body that may have a smooth or finely nodular surface. Anatomical variants (such as the bilobed) may themselves enlarge as a consequence of carotid body hyperplasia.

Human carotid bodies undergo histological changes in cardiopulmonary disease, especially in chronic obstructive lung disease and systemic hypertension.' Such abnormal glomera may be detected by their macroscopic appearances at necropsy. Contrary to popular belief, dissection and examination of carotid bodies at necropsy takes only a few minutes and may yield worthwhile additional morbid anatomical data in cases of heart and lung disease. In making a macroscopic assessment of them care must be taken to distinguish pathological changes from anatomical variations.

\section{Patients and methods}

We dissected out the carotid bodies in 100 consecutive subjects undergoing necropsy by the method we have described elsewhere. ${ }^{1}$ They were examined macroscopically to see what forms they had assumed as a result both of anatomical variation and of pathology. The incidence of the different variants was noted as were the variations in their positions based on the origin of the glomic arteries supplying them.

\section{Results}

The incidence of the different forms on either side is shown in table 1 . The positions of the carotid bodies are shown in table 2 . In each position the carotid body was bound to the principal underlying blood vessel named by the fibrous tissue of the ligament of Mayer. This ensheaths the sinus nerve and one or more of the glomic arteries that are up to $4 \mathrm{~mm}$ long and arise directly from the main parent artery. ${ }^{2}$ At its origin the main glomic artery is encircled by closely packed bands of thick elastic fibres that are derived from the parent vessel and not guarded by sphincters (as in the rat). The tiny glomic arteries that help to anchor the carotid bodies in the positions shown in table 2 have luminal diameters of only $200 \mu \mathrm{m}$ but are predominantly elastic, reminiscent of the baroreceptor carotid sinus. This elastic histological structure is maintained in the interlobular glomic arteries but is replaced by muscularisation in the intralobular glomic arterioles. ${ }^{3}$ Several forms were identified.

OvoID

In most cases the carotid bodies on both sides were single and ovoid, sometimes with a pointed apex

Table 1 Variation in form of carotid bodies in 100 subjects coming to necropsy (figures are given as percentages)

\begin{tabular}{lcc}
\hline Form & Right & Left \\
\hline Single ovoid & 83 & 86 \\
Double & 7 & 6 \\
Bilobed (including V form) & 9 & 7 \\
Leaf like & 1 & 1 \\
\hline
\end{tabular}

Table 2 Position of carotid bodies in 100 subjects coming to necropsy (figures are given as percentages)

\begin{tabular}{lcc}
\hline Position & Right & Left \\
\hline Bifurcation & 86 & 87 \\
External carotid artery & 7 & 9 \\
Carotid sinus & 5 & 2 \\
Pharyngeal artery & 2 & 2 \\
\hline
\end{tabular}




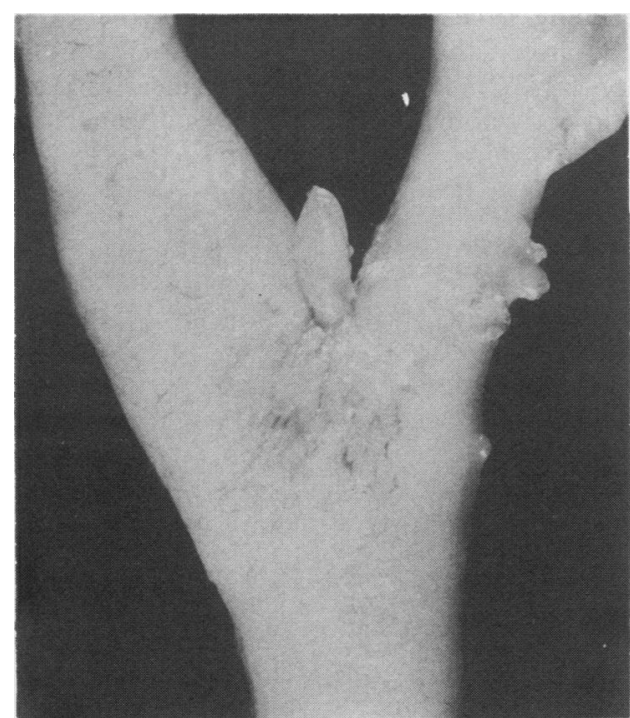

Fig 1 Normal ovoid left carotid body (weighing $8 \mathrm{mg}$ ) from a woman of 56 years with bronchial carcinoma; the right weighed $9 \mathrm{mg}$.

(fig 1), and situated at the bifurcation. They were usually sessile with the glomic artery hidden beneath them. Occasionally they were pedunculated, arising several millimetres along the glomic artery. This simple appearance is the classic form of the normal carotid body.

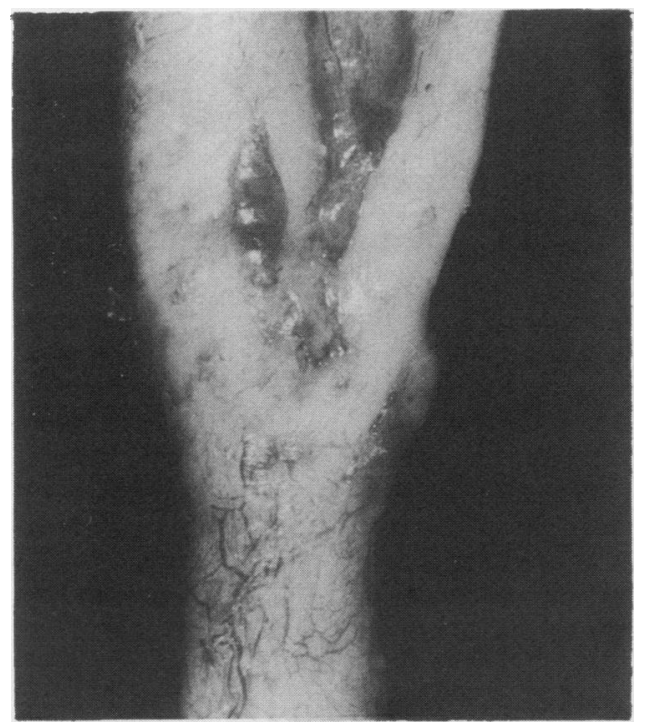

Fig 2 Double left carotid body from a woman of 61 years with coronary insufficiency. One (weighing $2.5 \mathrm{mg}$ ) is at the bifurcation and the other (weighing $4.5 \mathrm{mg}$ ) is over the left carotid sinus.

\section{DOUBLE}

In an appreciable minority double carotid bodies were found on one or other side, and they occurred in various places. Usually both members of the pair arose at the same site, such as the carotid bifurcation. Sometimes, however, they occurred at different sites, as in the case shown in fig 2, where one carotid body was present at the bifurcation and the other was supplied by a glomic artery arising from the carotid sinus. The combined weight of double carotid bodies was similar to that of normal single ones, because both members of the pair were roughly half the normal size.

\section{BILOBED}

In quite a large number of cases the carotid bodies were bilobed, two distinct apical lobes fusing at the base to share a common origin from a single glomic artery (fig 3). Bilobed carotid bodies were usually found at or near the bifurcation but occurred at any site where single bodies were found. In some instances the carotid bodies were V shaped (fig 4) but we regarded this as a variant of the bilobed variety rather than as a distinct form, the two lobes being fused to form a common base.

\section{LEAF-LIKE}

In two cases the carotid bodies on one or other side had assumed an expanded, leaf like appearance (fig 5). Double and bilobed forms accounted for $16 \%$ of carotid bodies on the right side and $13 \%$ on the left

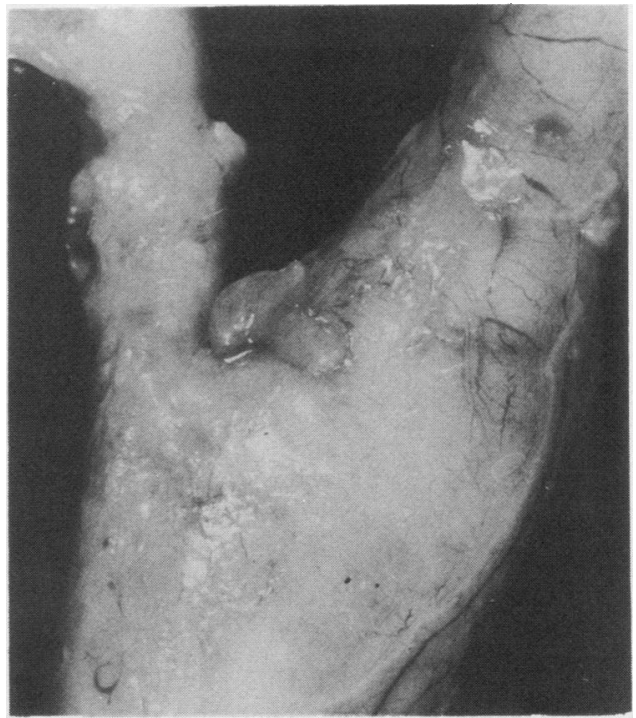

Fig 3 Bilobed right carotid body (weighing $15 \mathrm{mg}$ ) from a woman of 66 years with acute myeloid leukaemia. 
(table 1); the leaf-like form was found in only two cases, one on the right side and one on the left. In nearly all cases the carotid bodies were at the bifurcation and present in much smaller percentages, in

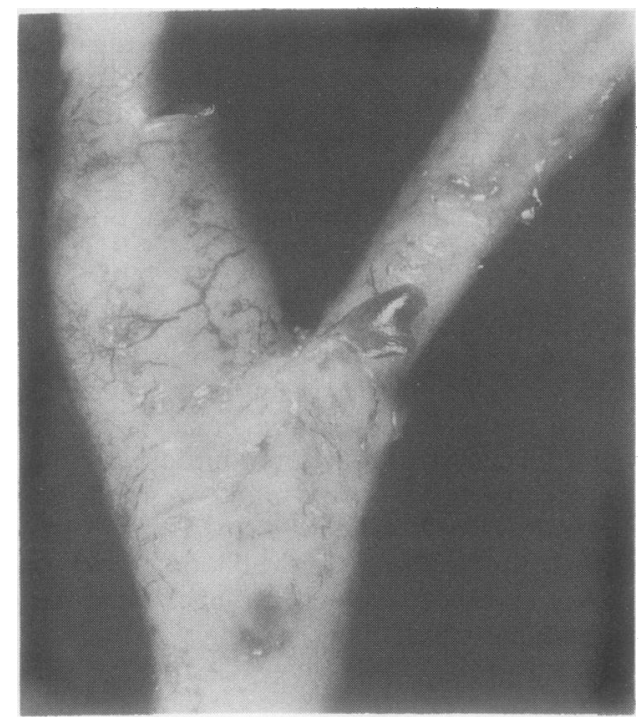

Fig $4 V$ shaped left carotid body (weighing $7 \mathrm{mg}$ ) arising from the bifurcation in a woman of 57 years dying from congestive cardiac failure caused by mitral stenosis.

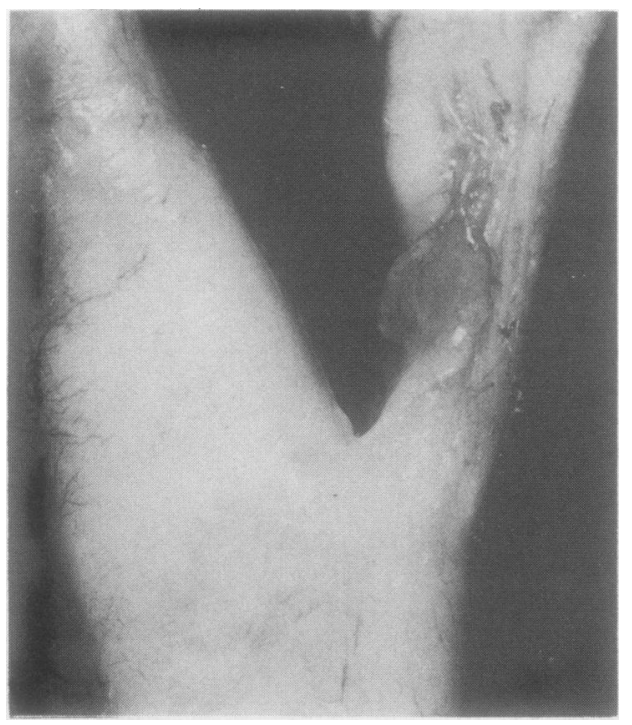

Fig 5 Leaf shaped left carotid body (weighing $14 \mathrm{mg}$ ) lying over left external carotid artery in a woman of 66 years with acute myeloid leukaemia. descending order of frequency, over the external carotid artery, the carotid sinus, and the pharyngeal artery (table 2). The different anatomical variants of carotid body noted in the 100 consecutive necropsies

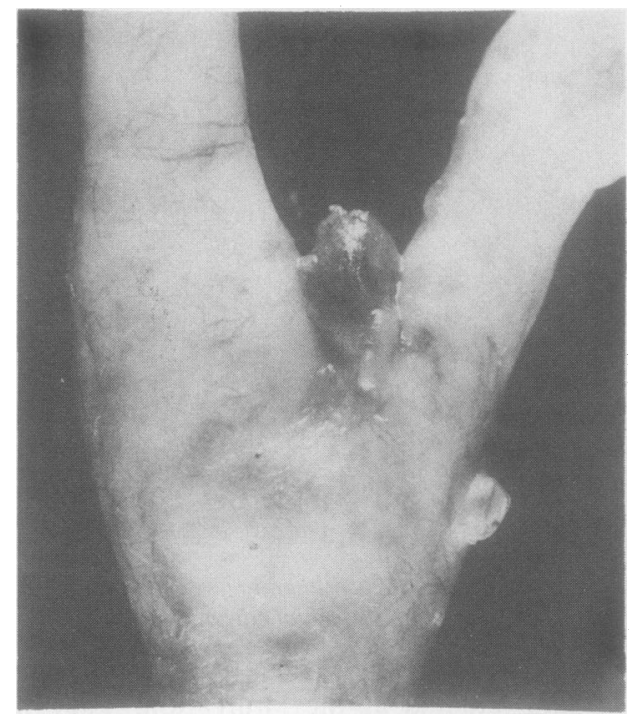

Fig 6 Left carotid body (combined carotid body weight $43.9 \mathrm{mg}$ ) from a man aged 62 years with panacinar emphysema and biventricular hypertrophy. It is large and ovoid with a smooth, cyanotic appearance.

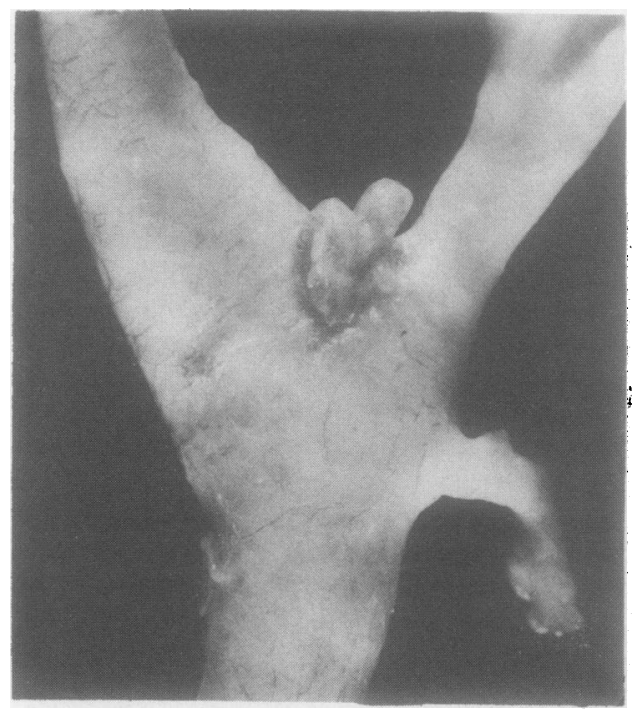

Fig 7 Left carotid body (combined carotid body weight $55.8 \mathrm{mg}$ ) from a man aged 58 years with pronounced left ventricular hypertrophy caused by systemic hypertension. It has an apparently coarse nodular appearance produced mainly by enlargement of a bilobed variant. 


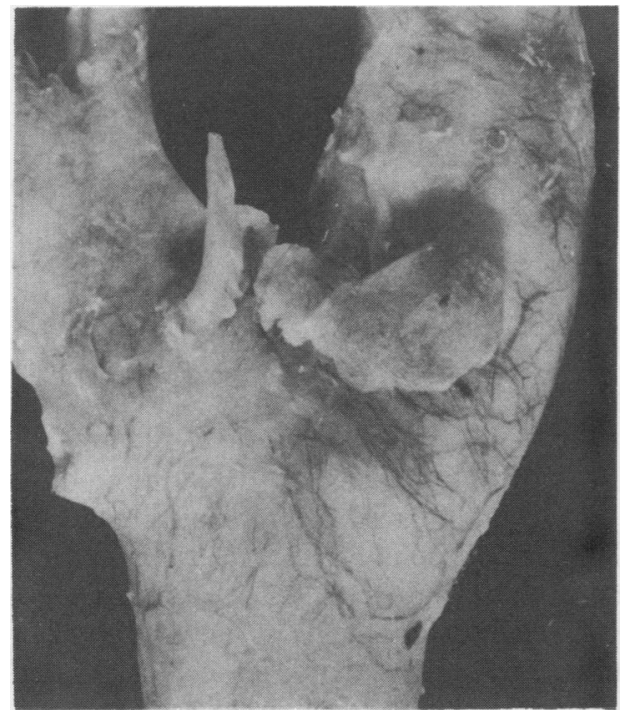

Fig 8 Right carotid bifurcation of a man of 53 years who had systemic hypertension and left ventricular hypertrophy. The carotid body arises from the bifurcation and is enlarged with fine nodularity. Microscopic examination showed that it was hyperplastic.

may be contrasted with pathological macroscopic changes in carotid bodies that we have reported previously. ${ }^{45}$

\section{ENLARGED OVOID}

In cases where there was histological evidence of carotid body hyperplasia associated with systemic hypertension or with pulmonary emphysema complicated by hypoxaemia the carotid bodies were unequivocally enlarged but retained the smooth, ovoid appearance (fig 6). The hypoxaemia caused the enlarged carotid bodies to have a cyanotic hue.

\section{COARSE NODULARITY}

In one case associated with systemic hypertension previously reported by $\mathrm{us}^{4}$ the carotid body showed what seemed to be a coarsely nodular appearance (fig 7). Closer examination, however, suggested that the two main nodules seemed to represent a bilobed organ. The smaller paler nodules seemed to be remaining tags of adipose tissue. We interpret such appearances as pathological enlargement with an anatomical variant. The carotid body on the other side showed uniform ovoid enlargement.

\section{FINE NODULARITY}

In this condition the carotid bodies were enlarged but finely nodular (fig 8), and histological examination showed sustentacular cell hyperplasia." Thus fine nodularity seems to represent a pathological change rather than an anatomical variant.

\section{Discussion}

Our study shows that when carotid bodies are examined at necropsy they may present a considerable range of macroscopic appearances. Some of these are anatomical variants but others are of pathological importance and it is necessary to distinguish between the two. The classic carotid body is a simple ovoid but it has several anatomical variants that are, in descending order of frequency, the bilobed (including the $\mathrm{V}$ form), the double, and the leaf shaped. The hallmark of pathological abnormality on macroscopic appearance is enlargement, which indicates histological hyperplasia. Usually the enlarged glomus retains its ovoid appearance with a smooth surface, but sometimes it assumes a fine nodularity. In cases of chronic obstructive lung disease it is cyanotic. Finally it has to be kept in mind that anatomical variants themselves may become hyperplastic so that enlargement of a bilobed or multilobed carotid body may lead to the macroscopic appearance of coarse nodularity.

The considerable degree of anatomical variation in both form and position of the carotid bodies in man is a reflection of that found in other mammals. Adams ${ }^{6}$ gives many examples of this in his exhaustive study of the comparative morphology of the carotid body and sinus.

We thank the editors of Thorax and the Journal of Pathology for permission to reproduce figs 6 and 7,4 and $8 .^{5}$

\section{References}

1 Heath D, Smith P. The pathology of the carotid body and sinus. London: Edward Arnold, 1985.

2 Jago R, Heath D, Smith P. Structure of the glomic arteries. $J$ Pathol 1982;138:205-18.

3 Heath D, Jago R, Smith P. The vasculature of the carotid body. Cardiovasc Res 1983;17:33-42.

4 Heath D, Edwards C, Harris P. Post-mortem size and structure of the human carotid body. Thorax 1970;25:129-40.

5 Smith P, Jago R, Heath D. Anatomical variation and quantitative histology of the normal and enlarged carotid body. J Pathol 1982;137:287-304.

6 Adams WE. The comparative morphology of the carotid body and carotid sinus. Springfield, Illinois: Charles C Thomas, 1958.

Requests for reprints to: Professor D Heath, Department of Pathology, Duncan Building, Royal Liverpool Hospital, PO Box 147, Liverpool L69 3BX, England. 